\title{
ICAT Promotes Cervical Cancer Cell Proliferation, Invasion, Migration and Epithelial-to-mesenchymal Transition through HPV16 E6, E7/ miR-23b-3p/ ICAT axis
}

\author{
Jing Hu \\ Chongqing Medical university \\ Zijiu Sun \\ Chongqing Medical University \\ Hui Wang \\ The First Affiliated Hospital of Chongqing Medical University \\ Wei Ren \\ The First Affiliated Hospital of Chongqing Medical University \\ Yuting Fang \\ Chongqing Medical University \\ Kai Hu \\ Chongqing Medical university \\ Huomei Yu \\ Chongqing Medical University \\ Deyu Liao \\ Chongqing Medical University \\ Shiyan Liu \\ Chongqing Medical university \\ Lan Zhou \\ Chongqing Medical University \\ Tongchuan He \\ University of Chicago Medical center \\ Yan Zhang ( $\square$ yanzhang@cqmu.edu.cn ) \\ Chongqing Medical University https://orcid.org/0000-0001-5413-4598
}

Primary research

Keywords: HPV 16 E6 E7, miR-23b-3p, ICAT, cervical cancer

Posted Date: November 5th, 2020

DOI: https://doi.org/10.21203/rs.3.rs-101315/v1

License: (c) (7) This work is licensed under a Creative Commons Attribution 4.0 International License. Read Full License 


\begin{abstract}
Background: Human papillomavirus (HPV) 16 plays a crucial role in cervical cancer (CC) development. Previous study reported that inhibitor of $\beta$-catenin and TCF (ICAT) is upregulated in CC and promotes cervical tumor progression. Herein, we aimed to investigate the underlying molecular mechanism that HPV16 regulates the expression of ICAT and promotes the CC development.

Methods: The expressions of HPV 16 E6, E7 and ICAT were modulated by small interfering RNA and recombinant adenovirus, respectively. qRT-PCR was conducted to detect the mRNA expression of HPV 16 E6, E7, ICAT and miR-23b-3p in SiHa and CasKi cells. Bioinformatics analysis was utilized to predict the potential miRNAs that could bind to the ICAT 3' untranslated region. Then, the dual luciferase reporter assay was used to confirm that. Cell proliferation ability was detected by CCK-8 assay. Wound healing and Transwell assays were used to observe migration and invasion abilities. Protein expressions were measured with western blot.

Results: Results revealed that after knocking down of HPV16 E6, E7, the expression of ICAT decreased, but the expression of miR-23b-3p increased. Besides, miR-23b-3p negatively modulated ICAT expression in HPV16 positive CC cells. Dual luciferase assays confirmed that ICAT was a target gene of miR-23b-3p. Functional experiments showed that the overexpression of miR-23b-3p suppressed malignant behaviors of SiHa and CasKi cells, such as migration, invasion and EMT. Importantly, the overexpression of ICAT counteracted the suppressive effect of miR-23b-3p on HPV16 positive cervical cancer cell. Furthermore, after the knockdown of HPV16 E6 and E7, the inhibition of miR-23b-3p could increase the ICAT expression and rescue the siRNA HPV16 E6, E7-mediated suppressive impact on the aggressiveness of SiHa and CasKi cells.
\end{abstract}

Conclusions: Our study demonstrates that HPV 16 E6, E7/miR-23b-3p/ ICAT axis plays an important role in HPV16 positive CC pathogenesis, which may serve as a promising therapy target for HPV 16-associated cervical cancer.

\title{
1 Introduction
}

Cervical cancer is identified as one of the most frequent malignancies in women ${ }^{1}$, with high incidence and mortality rate ${ }^{2}$. According to the statistics, there are more than 500,000 new patients diagnosed with cervical cancer and approximately 300,000 deaths estimated each year ${ }^{3}$. Persistent infection with high-risk human papillomavirus (HR-HPV) has been recognized as a key risk factor for cervical cancer ${ }^{4}$. Results from epidemiological studies have shown that HPV16 is the most important and common high-risk type of HPV, which accounts for over one half of cervical cancer cases ${ }^{5,6}$. HPV 16 E6 and E7, two key oncoproteins encoded by oncogenic HPV 16, can modulate many other multifunctional genes and downstream effectors and thus result in cervical cancer development ${ }^{7}$. HPV16 E6 induces the ubiquitination degradation of tumor suppressor p53 and, ultimately prevents cells from growth arrest and apoptosis ${ }^{8}$. While HPV16 E7 predominantly induces degradation of protein retinoblastoma (pRb), then releases E2F1, and eventually promotes cell proliferation ${ }^{9-11}$. However, the precise molecular mechanism of HPV 16 E6 and E7 mediated cervical cancer has not been completely elucidated.

The inhibitor of b-catenin and TCF (ICAT) is a small protein composed of 81 amino acids, which was first discovered in 2000 as a negative regulator of Wnt signaling pathway. Dysfunction of ICAT can regulate cancer progression that is involved in cell growth, apoptosis, invasion and metastasis. Previous studies have revealed that ICAT can restrain different human cancers, including colorectal cancer, lung adenocarcinomas, glioblastoma, so on ${ }^{12-14}$. Notably, our recent study demonstrated that ICAT could accelerate cell growth and metastasis by interrupting the E-cadherin/ $\beta$-catenin complex in cervical cancer ${ }^{15}$. However, the precise molecular mechanisms of ICAT-mediated cervical cancer are still limited.

MiRNAs are small non-coding RNAs and serve as a crucial regulator by conjugating the 3 '-untranslated region (3'-UTR) of target genes, modulating cervical cancer progression ${ }^{16,17}$. Previous study have demonstrated that miR-23b shows significantly reduced expression in cervical cancer ${ }^{18}$, and in this study bioinformatics analysis revealed that the 3 '-UTR of ICAT contained a potential binding site for miR-23b-3p. Furthermore, high-risk HPV E6 and E7 can manipulate the expression of miRNAs and then impact downstream targets expression, which are involved in various tumor development ${ }^{7,19}$. Therefore, it is hypothesized that HPV16 E6, E7 can regulate the expression of ICAT through miR-23b-3p, thereby promoting the proliferation, migration and metastasis of cervical cancer cells. The aim of this study is to provide a thorough understanding of the underlying molecular mechanism of HPV 16 E6,E7 and ICAT in the progression of cervical cancer, which may suggest a new insight into therapeutic approaches for cervical cancer.

\section{Materials And Methods}

\subsection{Cell Culture}

HPV16-positive (CaSki and SiHa cells) cervical cancer cells and HEK293T cells were purchased from American Type Culture Collection (ATCC, Manassas, VA, USA), maintained in Dulbecco's modified Eagle's medium (DMEM; HyClone Laboratories, Inc., Logan, uT, USA), and supplemented with $10 \%$ fetal bovine serum (FBS; Gibco, Carlsbad, CA, USA) with $100 \mathrm{U} / \mathrm{mL}$ penicillin and $100 \mathrm{mg} / \mathrm{mL}$ streptomycin at $37^{\circ} \mathrm{C}$ with $5 \% \mathrm{CO}_{2}$

\section{2 miRNA transfection, siRNA interference and adenoviral infection}

The miR-23b-3p mimic, anti-miR-23b-3p, and negative control were purchased from Shanghai GenePharma, Co., Ltd. (Shanghai, China). The E6, E7 siRNA and the corresponding negative control siGL3 were purchased from Biomics Biotechnologies, Co., Ltd. (Jiangsu, China). Their sequences are shown in Table 1. $\mathrm{SiHa}$ and CasKi cells were transfected using Lipofectamine ${ }^{\circledR} 2000$ (Invitrogen, CA, USA) according to the manufacturer's protocol. After 6-8 h of transfection, the medium was replaced with fresh growth medium. 
Recombinant adenovirus AdICAT and the corresponding negative control AdRFP were kindly donated by Dr Tongchuan He (university of Chicago Medical Center, Chicago, IL, USA). SiHa and CasKi cells were infected with AdICAT and AdRFP with polybrene (Sigma-Aldrich). The medium was replaced with fresh medium after $8 \mathrm{~h}$ of cultivation. Fluorescence was then observed after $36 \mathrm{~h}$.

Table 1

Primers and siRNA sequences.

\begin{tabular}{|c|c|c|}
\hline Targets & & Sequence \\
\hline \multirow[t]{2}{*}{ si16E6, E7 } & Forward & 5'-GAC CGG UCG AUG UAU GUC UUG-3 \\
\hline & Reverse & 5'-AGA CAU ACA UCG ACC GGU CCA-3' \\
\hline \multirow[t]{2}{*}{ siGL3 } & Forward & 5'-CUU ACG CUG AGU ACU UCG-3' \\
\hline & Reverse & 5'-UCG AAG UAC UCA GCG UAA-3' \\
\hline \multirow[t]{2}{*}{ ICAT } & Forward & 5'-ATGAACCG CGAGGGAGCTCC-3' \\
\hline & Reverse & 5'-CTACTGCCTCCG GTCTTCCG-3' \\
\hline \multirow[t]{2}{*}{ E6, E7 } & Forward & 5'-CAATGTTTCAGGACCCACAGG-3' \\
\hline & Reverse & 5'-CTCACGTCGCAG-TAACTGTTG-3' \\
\hline \multirow[t]{2}{*}{ miR-23b-3p } & Forward & 5'-GAGCATCACATTGCCAGGG-3' \\
\hline & Reverse & 5'-GTGCAGGGTCCGAGGT-3' \\
\hline \multirow[t]{2}{*}{ GAPDH } & Forward & 5'-GCACCGTCAAGGCTGAGAAC-3' \\
\hline & Reverse & 5'-TGGTGAAGACGCCAGTGGA-3' \\
\hline \multirow[t]{2}{*}{ U6 } & Forward & 5'-GCGCGTCGTGAAGCGTTC-3' \\
\hline & Reverse & 5'-GTGCAGGGTCCGAGGT-3' \\
\hline
\end{tabular}

\subsection{Cell counting kit-8 (CCK8) assay}

A Cell Counting kit-8 assay kit (CCK-8; Beyotime Institute of Biotechnology, Haimen, China) was used to evaluate cell proliferation ability. $24 \mathrm{~h}$ after transfection, cells were resuspended and plated into the 96 -well plate at a density of 3000 cells/well in $100 \mu$ l complete DMEM with $10 \%$ FBS. Cells were cultured in an incubator at $37^{\circ} \mathrm{C}$ with $5 \% \mathrm{CO}_{2}, 10 \mu \mathrm{l}$ of CCK-8 reagent was added into each well of the plate at $12,24,48,72$ and $96 \mathrm{~h}$. Subsequent to incubation at $37^{\circ} \mathrm{C}$ for another $2 \mathrm{~h}$, the absorbance (OD value) of each well was measured at $450 \mathrm{~nm}$ using a microplate reader (Bio-Rad Laboratories, Inc., Hercules, CA, USA).

\subsection{Wound healing assay}

The transfected $\mathrm{SiHa}$ and CasKi cells were seeded in a 6-well plate and incubated at $37^{\circ} \mathrm{C}$ until they were at least $90 \%$ confluent. A wound field was made using a sterile 10- $\mu$ lip among cells in each well. After washed with phosphate-buffered saline (PBS) three times, 2 mL of serum-free DMEM was added. Wound healing was observed under an inverted phase contrast microscope, and images were captured at 0,24 and $48 \mathrm{~h}$ after the wound was made. The wounding healing rate was evaluated as: (W1-W2)/W1× $100 \%$, where W1 is the Oh wound width and W2 is the $24 \mathrm{~h}$ and $48 \mathrm{~h}$ wound width. This assay was independently repeated three times.

\subsection{Transwell migration and invasion assays}

The cell migration and invasion assays were designed in Transwell chambers (24-well Transwell chambers, 8- $\mu \mathrm{m}$ pore size; Corning, Inc., Corning, NY, USA). In the migration assay, $5 \times 10^{4}$ transfected cells were suspended in serum-free medium and added into the upper chamber. In the invasion assays, $8 \times 10^{4}$ transfected cells in serum-free medium were seeded into the upper chamber of the Transwell after diluted Matrigel was added. Medium containing $20 \%$ FBS was added into the lower chamber and used as a chemoattractant. After $24 \mathrm{~h}$ incubation at $37^{\circ} \mathrm{C}$ of a $5 \% \mathrm{CO}_{2}$ atmosphere, the Transwell chambers were taken out, fixed with $4 \%$ paraformaldehyde for $15 \mathrm{~min}$ and stained with $0.1 \%$ crystal violet for $10 \mathrm{~min}$ at room temperature. The cells on the upper surface of the chamber that did not pass through were carefully removed using a cotton swab. Then the cells were counted and photographed by Inversion Microscope, and each group was counted for 5 fields of view. The ImageJ software was used to cell count. Each assay was carried out in triplicate.

\subsection{RNA isolation and real-time quantitative RT-PCR analysis (qRT-PCR)}

Total RNA from cervical cancer cells were isolated using TRIzol reagent (Invitrogen, Carlsbad, CA, USA) according to the manufacturer's instructions. Total RNA $(1 \mu \mathrm{g})$ was used for cDNA synthesis using an RNA reverse transcription amplification kit (TaKaRa). A real-time quantitative polymerase chain reaction (qRTPCR) assay was performed to evaluate mRNA and mature miRNA expression using the SYBR-Green PCR Master Mix (TaKaRa). The target gene expression Loading [MathJax]/jax/output/CommonHTML/jax.js . Primers used in this study were shown in Table 1 


\subsection{Western Blot Analysis}

$48 \mathrm{~h}$ post-transfection, proteins were extracted from cells using a radioimmunoprecipitation lysis buffer (Beyotime Institute of Biotechnology, Shanghai, China). Protein concentrations were measured using a BCA Protein Assay kit (Beyotime Institute of Biotechnology, Shanghai, China). After denaturation in boiling water, equal amount of proteins was loaded, separated by $8-15 \%$ sodium dodecyl sulphate-polyacrylamide gel electrophoresis (SDS-PAGE) and then transferred onto PVDF membranes. The membranes were blocked with $5 \%$ bovine serum albumin at $37^{\circ} \mathrm{C}$ for $2 \mathrm{~h}$. The membranes were washed with TBST and incubated at $4^{\circ} \mathrm{C}$ overnight with respective primary antibody against E-cadherin, N-cadherin, Vimentin (1:1000; Cell Signaling Technology, Danvers, MA, USA), PCNA, Cyclin D1 (1:1000; Wanleibio Co., Ltd., Beijing, China), ICAT (1:1,000; Abcam, Cambridge, UK), and $\beta$-actin (1:1000; Santa Cruz Biotechnology, CA, USA). After that, the membrane was washed using TBST for 3 times/10 min and incubated with a horseradish peroxidase (HRP)-labeled secondary antibody (1:5,000; Beijing Zhongshan Golden Bridge Biotechnology) for $1 \mathrm{~h}$ at $37^{\circ} \mathrm{C}$. Next the membrane was washed with TBST for 3 times $/ 10$ min. This was followed by treatment with HRP-conjugated secondary antibody (1:5000; Beijing Zhongshan Golden Bridge Biotechnology) for $1 \mathrm{~h}$ at $37 \circ \mathrm{C}$. The protein bands were visualized with the Supersignal West Pico Chemiluminescent substrate kit (Millipore, Billerica, MA, USA) and $\beta$-actin was used as an internal control. All experiments were performed in triplicate.

\subsection{Bioinformatics analysis and dual luciferase reporter assay.}

According to the results obtained from the online prediction system, TargetScan (http://www.targetscan.org), miRBase (http://www.mirbase.org/), and miR23b-3p was predicted as miRNAs that could potentially regulate ICAT. It was shown in the databases that there was a potential binding sequence in the 3'untranslated region (UTR) of ICAT mRNA that matched with miR-23b-3p. The sequence fragments in the $3^{\prime}$-UTR of target gene ICAT that complementarily bind to miR-23b-3p and mutant (mut) ICAT 3'-UTR sequence fragments were synthesized and cloned into a pGL6 vector. For the dual-luciferase reporter assays, HEK293T cells were first cultured in a 24-well culture plate $\left(10 \times 10^{4}\right.$ cells/well). Then, the correctly sequenced luciferase reporter plasmids WT or MUT were respectively co-transfected with miR-23b-3p mimic or inhibitor and pRL-TK plasmid coding Renila luciferase into the HEK-293T cells. The cells were collected at $48 \mathrm{~h}$ after transfection to determine the luciferase activity using a Dual-Luciferase reporter gene assay kit (Promega, Madison, WI, USA), adhering to manufacturer's protocol. Firefly luciferase activity was normalized against the activity of the Renilla luciferase gene.

\subsection{Statistical Analysis}

Data were expressed as the mean \pm standard deviation of at least three independent experiments. GraphPad Prism 8.3.0 software (GraphPad Software Inc., La Jolla, CA, USA) was used for statistical analysis. Data were analyzed by using Student's t-test or ANOVA to assess the statistical significance. P<0.05 was considered to indicate a statistically significant difference.

\section{Results}

\subsection{Knockdown of HPV16 E6, E7 decreased ICAT but increased miR-23b-3p expression}

The constant expression of High-risk HPV16 E6, E7 are considered to be the crucial cause of cervical cancer, and our previous study showed that ICAT was highly expressed in cervical cancer. Herein, we hypothesized the up-regulated expression of ICAT was associated with HPV16 E6, E7. qRT-PCR analysis showed the mRNA levels of E6 and E7 were suppressed in cells treated with the HPV16 E6, E7 small interfering RNA (siRNA) (Figure 1A). Compared with control, the mRNA and protein levels of ICAT were significantly down-regulated in SiHa cells where HPV16 E6, E7 were down-regulated. Similar results were also found in CasKi cells (Figure 1B and 1C). Taken together, the results indicated that HPV16 E6, E7 could regulated the expression of ICAT.

Previous studies has documented that High-risk HPV16 E6, E7 can cause alterations in the expression of microRNAs, and another study confirmed that miR23b shows significantly reduced expression in cervical cancer ${ }^{18}$. To determine the possible interaction between HPV16 E6, E7 and miR-23b-3p, the expression level of miR-23b-3p in SiHa and CasKi cells was validated by qRT-PCR after transfected with HPV16 E6, E7 siRNA. As expected, knockdown of HPV16 E6, E7 increased miR-23b-3p expression in the two HPV16 positive cervical cancer cell lines, SiHa and CaSki (Figure 1D).

\section{2 miR-23b-3p repressed ICAT expression in SiHa and CasKi cells}

As the expression of miR-23b-3p was inversely proportionate to that of ICAT after HPV16 E6, E7 were knocked down in cervical cancer cells, further research was needed to determine whether miR-23b-3p can affect the expression of ICAT in SiHa and CasKi cells. To verify the hypothesis, miR-23b-3p mimic, miR-23b$3 p$ inhibitor or a negative control were transfected into the $\mathrm{SiHa}$ and CasKi cells (Figure 2A). qRT-PCR and western blot analysis revealed that the expression levels of ICAT mRNA and protein in the SiHa and CasKi cells transfected with miR-23b-3p mimic were lower than those transfected with the control group. Consistently, results demonstrated that the mRNA and protein expression of ICAT in SiHa and CasKi cells transfected with miR-23b-3p inhibitor was significantly upregulated compared with the negative control (Figure $2 \mathrm{~B}$ and $2 \mathrm{C}$ ). Accordingly, these findings indicate that miR-23b-3p can negatively regulate the expression of ICAT in HPV16 positive cervical cancer cells.

\section{3 miR-23b-3p targeted the 3'-UTR of ICAT mRNA}

Loading [MathJax]/jax/output/CommonHTML/jax.js

Page $4 / 15$ 
To further investigate whether there is a direct interaction between miR-23b-3p and ICAT gene, bioinformatic analysis was performed with TargetScanHuman 7.2 and miRBase database. Results revealed that the 3 '-UTR of ICAT contained a potential binding site for miR-23b-3p (Figure 3A). Whereafter, a dual luciferase reporter assay was carried out to verify the prediction. The results of luciferase reporter assay demonstrated that the luciferase activity remarkably decreased compared to the mutant control when HEK 239 cells were co-transfected with miR-23b-3p mimic. In contrast, the activity increased compared to the negative control after HEK 239 cells were co-transfected with miR-23b-3p inhibitor (Figure 3B and 3C). Accordingly, these results demonstrate that ICAT is a novel target gene of miR-23b-3p.

\section{4 miR-23b-3p suppresses proliferation, migration, invasion and epithelial-mesenchymal transition (EMT) of cervical carcinoma cell}

To confirm whether miR-23b-3p affects HPV16 positive cervical cancer cell proliferation, CCK-8 and Western blot assays were performed. The results of CCK-8 tests revealed that the proliferation of cells transfected with miR-23b-3p mimics was significantly lower than that of the vector groups at $48 \mathrm{~h}$, $72 \mathrm{~h}$, and $96 \mathrm{~h}$, while the opposite results were observed in cells transfected with miR-23b-3p inhibitor (Figure 4A). Furthermore, we detected the protein levels of PCNA and Cyclin D1 by western blot. The results showed that the expressions of PCNA and Cyclin D1 were downregulated after cells were treated with miR-23b-3p mimic. However, the treatment with miR-23b-3p inhibitor caused increased expression of PCNA and cyclin D1(Figure 4B).

Cell migration and invasion are considered to be key events in tumor progression. Thus, wound healing assay and transwell assay were further performed to ascertain whether miR-23b-3p is involved in HPV16 positive cervical cancer cells migration and invasion. The results revealed that the overexpression of miR23b-3p significantly impaired the migration and invasion abilities of CasKi and SiHa cells, while the miR-23b-3p inhibitor markedly improved the abilities of migration and invasion in CaSki and SiHa cells (Figure 4C and 4D).

EMT is demonstrated to be a critical step in the metastatic cascade. To future investigate whether miR-23b-3p suppressed cell migration and invasion through inhibition of EMT, we measured the expressions of related markers by western blot. The results showed that the expression of E-cadherin increased in response to the overexpression of miR-23b-3p, accompanied by decreased expression of $\mathrm{N}$-cadherin and vimentin, and vice versa (Figure 4E).

Taken together, these data suggest that miR-23b-3p inhibits HPV16 positive cervical cancer cells proliferation, invasion, migration and EMT.

\subsection{ICAT overexpression reversed the antitumor impact of miR-23b-3p}

Since ICAT was found to be the direct target of miR-23b-3p, we further verified whether the miR-23b-3p impairs the progression of cervical carcinoma cells via regulating ICAT. Hence, miR-23b-3p mimic + AdRFP and miR-23b-3p mimic + AdICAT were co-transfected into SiHa and CasKi cells. Western blot analysis showed that the expression of ICAT was significantly up-regulated in cells transfected with the AdICAT (Figure 5B). CCK-8 and western blot assays showed that the effect of miR-23b-3p on the proliferation of SiHa and CasKi cells was inhibited by overregulation of ICAT (Figure 5A and 5B). Migration and invasion assays showed that the cellular migratory and invasive abilities were strikingly reserved by co-transfection with miR-23b-3p and ICAT in cells (Figure $5 \mathrm{C}$ and 5D). Furthermore, western blot data showed that overexpression of ICAT significantly rescued the effect of miR-23b-3p on the EMT in the SiHa and CasKi cells (Figure 5E). Above results demonstrate that ICAT overexpression partially reverse the inhibitory effects of miR-23b-3p on the HPV16 positive cervical cancer cells.

\subsection{HPV16 E6, E7 regulated the progression of cervical carcinoma cell through miR-23b- $3 p$ and ICAT}

HPV16 E6, E7 are documented to be important oncoproteins in cervical carcinoma. To further verify whether miR-23b-3p and ICAT were involved in the effect of HPV16 E6, E7 on the progression of cervical cancer cells, SiHa and CasKi cells were co-transfected HPV16 E6, E7 siRNA with miR-23b-3p inhibitor and inhibitor NC respectively. CCK-8 and western blot assays revealed that knockdown of miR-23b-3p could reverse the inhibition effects of siRNA HPV16 E6, E7 on proliferation of cervical cancer cells (Figure $6 \mathrm{~A}$ and $6 \mathrm{~B}$ ). Similar results were also observed in wound healing and transwell assays, that reduced migration and invasion abilities induced by HPV16 E6, E7 knockdown could be rescued by miR-23b-3p inhibitor (Figure 6C and 6D). Besides, co-transfected with miR-23b-3p inhibitor could decrease siRNA HPV16 E6, E7-mediated down-regulation of N-cadherin and Vimentin expressions and could reverse siRNA HPV16 E6, E7mediated upregulation of E-cadherin expression (Figure 6E). Furthermore, western blot analysis showed that the protein expression of ICAT was significantly increased in the HPV16 E6, E7 + miR-23b-3p inhibitor group compared with that in the control group (Figure 6B), indicating that HPV16 E6, E7 regulated ICAT via miR-23b-3p. These results demonstrate that HPV16 E6, E7 can regulate ICAT via miR-23b-3p and affect the progression of cervical cancer cells.

\section{Discussion}

Cervical cancer is the second most frequent malignant carcinoma in female around the world and more than $99 \%$ of cervical carcinomas are associated with High-risk HPVs ${ }^{20,21}$. Although surgical or chemotherapy treatments have significantly improved in recent years, tumor recurrence and metastasis remain high, which are the main causes of the unfavorable prognosis for CC patients ${ }^{22}$. Therefore, it is urgent to explore the underlying molecular mechanisms of HPV in the progression of cervical cancer. In the present study, we found HPV16 E6, E7 could regulated the expression of ICAT, which can mediate the proliferation, migration, invasion, and EMT progression of cervical cancer cells through miR-23b-3p. Thus, the HPV16 E6, E7/miR-233b-3p/ICAT axis can be used as a new potential therapeutic approach for HPV 16 positive cervical cancer.

Loading [MathJax]/jax/output/CommonHTML/jax.js 
High-risk HPVs are a large group of small DNA tumor viruses which is a crucial factor for the cervical cancer development. Despite that the HPV vaccines are available to prevent the HPV16 or 18 infections, it is essential to explore the in-depth mechanism for how HPVs cause cancer, since the vaccines do not remove pre-existing HPV infection or cure the HPV-related cancer patients. HPV16 is the most common type of high-risk HPV infection in cervical cancer. E6 and E7 are the major oncoproteins in tumorigenesis. Several reports have demonstrated that E6 and E7 can modulate the host cell gene expression. For example, HPV16 E6 expression causes mTORC1 activation as a result of increased AKT activity, and silencing E6 expression in cervical cells resulted in loss of active STAT3 ${ }^{23,24}$. Additionally, Rong F et.al reported that overexpression of E6 and E7 upregulated GLUT1 expression in lung cancer ${ }^{25}$. Our previous study demonstrated that ICAT was highly expressed in cervical cancer tissues and promoted cell proliferation, migration, invasion, and EMT processes. In order to investigate whether the aberrant expression of ICAT in CC is associated with E6 and E7, siRNA was applied to knockdown the expression of HPV 16 E6, E7 in $\mathrm{SiHa}$ and CasKi cell lines. Our study found that silencing HPV 16 E6, E7 significantly reduced the expression of ICAT both of mRNA and protein. These results indicate that the HPV 16 E6, E7 can regulate the expression of ICAT.

Accumulating evidence has shown that miRNAs can silence the expression of target genes and then influence the cell differentiation, proliferation, migration, apoptosis and invasion ${ }^{26}$. Notably, it is demonstrated that HPV 16 E6, E7 can regulate the expression of cellular miRNAs and then alter gene expression, which may accelerate the development of the tumor ${ }^{7}$. miR-23b-3p is first discovered as a tumor suppressor whose expression is down-regulated in several cancers, such as colon cancer, prostate cancer, human hepatocellular carcinoma ${ }^{27-29}$, etc. What's more, the researchers find that miR-23b demonstrate significantly reduced abundance in cervical cancer cell lines compared with normal cervix ${ }^{18,30}$. To verify whether the abnormal expression of miR-23b-3p is associated with HPV 16 E6, E7, the relative expression of miR-23b-3p was detected after the expression of HPV 16 E6, E7 was silenced. Our results showed that inhibiting HPV 16 E6, E7 significantly up-regulated the expression of miR-23b-3p in SiHa and CasKi cells, which is consistent with the previous report ${ }^{31}$. Furthermore, we analyze ICAT as a potential target gene of miR-23b-3p by using several bioinformatics databases and performing dual luciferase reporter assay to verify that. Besides, transfecting with miR-23b-3p mimic repressed ICAT expression. On the contrary, inhibition of miR-23b-3p significantly upregulated the ICAT expression. To further understanding the mechanism involved, SiHa and CasKi cells were co-transfected HPV16 E6, E7 siRNA with miR-23b-3p inhibitor and inhibitor NC, respectively. The results showed that transfecting with miR-23b-3p inhibitor counteracted the repression effects of HPV16 E6, E7 siRNA on ICAT expression. Therefore, the present data demonstrate that HPV16 E6, E7 can regulate ICAT via miR-23b-3p.

Previous study have reported that ICAT is highly expressed in cervical cancer and can promote tumor cell proliferation, migration and invasion ${ }^{15}$. In this study, we observed that overexpression of the miR-23b-3p inhibited the cervical tumor cell progression, whereas the overexpression of ICAT counteracted the inhibitory effects. Subsequently, our results showed that knocking down the expression of miR-23b-3p in SiHa and CasKi cells could increase ICAT expression and promote cell proliferation, migration and invasion after HPV 16 E6, E7 was silenced. Metastatic toward adjacent and distal organs are responsible for the major threaten of cervical cancer related deaths. The epithelial-to-mesenchymal transition processes, characterized by the loss of epithelial protein E-cadherin and the increase of mesenchymal proteins $\mathrm{N}$-cadherin and Vimentin, are reported to play crucial roles in tumor progression ${ }^{32}$. In this study, the results showed that the overexpression of miR-23b-3p in SiHa and Caski cells decreased the expression of E-cadherin but increased that of $\mathrm{N}$-cadherin. However, the overexpression of ICAT strongly offset the effect of miR-23b-3p. In addition, our results also confirmed that reducing the expression of miR-23b-3p counteracted the effects of HPV 16 E6, E7 siRNA, which is reflected by the increased N-cadherin and, Vimentin expression and decreased E-cadherin expression. Consequently, these data verified that HPV16 E6, E7 could regulate ICAT via miR-23b-3p and affect the proliferation, migration, invasion and EMT of cervical cancer cells (Fig. 7).

\section{Conclusions}

our results demonstrate the that HPV 16 E6, E7 oncogenes possess the potential to regulate ICAT via miR-23b-3p and affect the progression of cervical cancer cells. This study might provide new insights into the role of HPV 16 E6, E7 in the development for cervical carcinoma, and might provide a promising therapeutic strategy against cervical cancer.

\section{List Of Abbreviations}

3'-untranslated region, 3'-UTR

adenovirus, Ad

cervical cancer, $\mathrm{CC}$

Cell Counting kit-8 assay, CCK-8

epithelial-mesenchymal transition, EMT

Human papillomavirus, HPV

high-risk human papillomavirus, HR-HPV

inhibitor of b-catenin and TCF, ICAT

protein retinoblastoma, $\mathrm{pRb}$

real-time quantitative polymerase chain reaction, qRT-PCR

Loading [MathJax]/jax/output/CommonHTML/jax.js 
small interfering RNA, siRNA

sodium dodecyl sulphate-polyacrylamide gel electrophoresis, SDS-PAGE

\section{Declarations}

\section{Ethics approval and consent to participate:}

Not applicable

\section{Consent for publication:}

Not applicable

\section{Availability of data and materials:}

All data generated or analysed during this study are included in this published article.

\section{Competing interests:}

The authors declare that they have no competing interests.

\section{Funding:}

This work was supported by National Natural Science Foundation of China (No. 81974449).

\section{Authors' contributions:}

$\mathrm{JH}, \mathrm{ZJS}$ and TCH analyzed the data and drew figures, JH and ZJS were the major contributors in writing the manuscript. HW, WR, YZ and YTF designed this work and were responsible for qRT-PCR, cck-8 assay and transwell assay. KH, HMY and DL performed the cell culture and western blot assay. SYL and LZ performed the dual luciferase reporter assay and wound healing assay. All authors read and approved the final manuscript.

\section{Acknowledgements:}

Not applicable

\section{References}

1. Jelastopulu E, Fafliora E, Plota A, et al. Knowledge, behaviours and attitudes regarding HPV infection and its prevention in female students in West Greece. European review for medical and pharmacological sciences 2016; 20(12): 2622-9.

2. Siegel RL, Miller KD, Jemal A. Cancer statistics, 2019. CA: a cancer journal for clinicians 2019; 69(1): 7-34.

3. Bray F, Ferlay J, Soerjomataram I, Siegel RL, Torre LA, Jemal A. Global cancer statistics 2018: GLOBOCAN estimates of incidence and mortality worldwide for 36 cancers in 185 countries. CA: a cancer journal for clinicians 2018; 68(6): 394-424.

4. Jimenez-Wences H, Peralta-Zaragoza O, Fernandez-Tilapa G. Human papilloma virus, DNA methylation and microRNA expression in cervical cancer (Review). Oncology reports 2014; 31(6): 2467-76.

5. Jalal Kiani S, Shatizadeh Malekshahi S, Yousefi Ghalejoogh Z, et al. Detection and Typing of Human Papilloma Viruses by Nested Multiplex Polymerase Chain Reaction Assay in Cervical Cancer. Jundishapur journal of microbiology 2015; 8(12): e26441.

6. Chen Q, Du H, Pretorius RG, et al. High-Grade Cervical Intraepithelial Neoplasia Detected by Colposcopy-Directed or Random Biopsy Relative to Age, Cytology, Human Papillomavirus 16, and Lesion Size. Journal of lower genital tract disease 2016; 20(3): 207-12.

7. Harden ME, Prasad N, Griffiths A, Munger K. Modulation of microRNA-mRNA Target Pairs by Human Papillomavirus 16 Oncoproteins. mBio 2017; 8(1).

8. Scheffner M, Huibregtse JM, Vierstra RD, Howley PM. The HPV-16 E6 and E6-AP complex functions as a ubiquitin-protein ligase in the ubiquitination of p53. Cell 1993; 75(3): 495-505.

9. Cobrinik D. Pocket proteins and cell cycle control. Oncogene 2005; 24(17): 2796-809.

10. Bernard $X$, Robinson $P$, Nomine $Y$, et al. Proteasomal degradation of $p 53$ by human papillomavirus E6 oncoprotein relies on the structural integrity of $p 53$ core domain. PloS one 2011; 6(10): e25981.

11. Gunasekharan V, Laimins LA. Human papillomaviruses modulate microRNA 145 expression to directly control genome amplification. Journal of virology 2013; 87(10): 6037-43 
12. Kang DW, Lee BH, Suh YA, et al. Phospholipase D1 Inhibition Linked to Upregulation of ICAT Blocks Colorectal Cancer Growth Hyperactivated by Wnt/beta-Catenin and PI3K/Akt Signaling. Clinical cancer research : an official journal of the American Association for Cancer Research 2017; 23(23): 7340-50.

13. Qi W, Chen J, Cheng X, et al. Targeting the Wnt-Regulatory Protein CTNNBIP1 by microRNA-214 Enhances the Stemness and Self-Renewal of Cancer StemLike Cells in Lung Adenocarcinomas. Stem cells 2015; 33(12): 3423-36.

14. Zhang K, Zhu S, Liu Y, et al. ICAT inhibits glioblastoma cell proliferation by suppressing Wnt/beta-catenin activity. Cancer letters 2015; $357(1)$ : 404-11.

15. Jiang Y, Ren W, Wang W, et al. Inhibitor of beta-catenin and TCF (ICAT) promotes cervical cancer growth and metastasis by disrupting E-cadherin/betacatenin complex. Oncology reports 2017; 38(5): 2597-606.

16. He Y, Lin J, Ding Y, et al. A systematic study on dysregulated microRNAs in cervical cancer development. International journal of cancer 2016; 138(6): 1312-27.

17. Li J, Liu Q, Clark LH, Qiu H, Bae-Jump VL, Zhou C. Deregulated miRNAs in human cervical cancer: functional importance and potential clinical use. Future oncology 2017; 13(8): 743-53.

18. Lui WO, Pourmand N, Patterson BK, Fire A. Patterns of known and novel small RNAs in human cervical cancer. Cancer research 2007; 67(13): 6031-43.

19. Yablonska S, Hoskins EE, Wells SI, Khan SA. Identification of miRNAs dysregulated in human foreskin keratinocytes (HFKs) expressing the human papillomavirus (HPV) Type 16 E6 and E7 oncoproteins. MicroRNA 2013; 2(1): 2-13.

20. Chung IH, Wu TI, Liao CJ, et al. Overexpression of lipocalin 2 in human cervical cancer enhances tumor invasion. Oncotarget 2016; 7(10): 11113-26.

21. McLaughlin-Drubin ME, Munger K. Oncogenic activities of human papillomaviruses. Virus research 2009; 143(2): $195-208$.

22. Small W, Jr., Bacon MA, Bajaj A, et al. Cervical cancer: A global health crisis. Cancer 2017; 123(13): 2404-12.

23. Shishodia G, Verma G, Srivastava Y, Mehrotra R, Das BC, Bharti AC. Deregulation of microRNAs Let-7a and miR-21 mediate aberrant STAT3 signaling during human papillomavirus-induced cervical carcinogenesis: role of E6 oncoprotein. BMC cancer 2014; 14: 996.

24. Spangle JM, Munger K. The human papillomavirus type 16 E6 oncoprotein activates mTORC1 signaling and increases protein synthesis. Journal of virology 2010; 84(18): 9398-407.

25. Fan R, Hou WJ, Zhao YJ, et al. Overexpression of HPV16 E6/E7 mediated HIF-1a upregulation of GLUT1 expression in lung cancer cells. Tumour biology : the journal of the International Society for Oncodevelopmental Biology and Medicine 2016; 37(4): 4655-63.

26. Gu J, Wang Y, Wu X. MicroRNA in the pathogenesis and prognosis of esophageal cancer. Current pharmaceutical design 2013; 19 (7): $1292-300$.

27. Zhang H, Hao Y, Yang J, et al. Genome-wide functional screening of miR-23b as a pleiotropic modulator suppressing cancer metastasis. Nature communications 2011; 2: 554.

28. Goto Y, Kojima S, Nishikawa R, et al. The microRNA-23b/27b/24-1 cluster is a disease progression marker and tumor suppressor in prostate cancer. Oncotarget 2014; 5(17): 7748-59.

29. Salvi A, Sabelli C, Moncini S, et al. MicroRNA-23b mediates urokinase and c-met downmodulation and a decreased migration of human hepatocellular carcinoma cells. The FEBS journal 2009; 276(11): 2966-82.

30. Shishodia G, Verma G, Das BC, Bharti AC. miRNA as viral transcription tuners in HPV-mediated cervical carcinogenesis. Frontiers in bioscience (Scholar edition) 2018; 10: 21-47.

31. Yeung CL, Tsang TY, Yau PL, Kwok TT. Human papillomavirus type 16 E6 suppresses microRNA-23b expression in human cervical cancer cells through DNA methylation of the host gene C9orf3. Oncotarget 2017; 8(7): 12158-73.

32. Frisch SM, Schaller M, Cieply B. Mechanisms that link the oncogenic epithelial-mesenchymal transition to suppression of anoikis. Journal of cell science 2013; 126(Pt 1): 21-9.

\section{Figures}


A

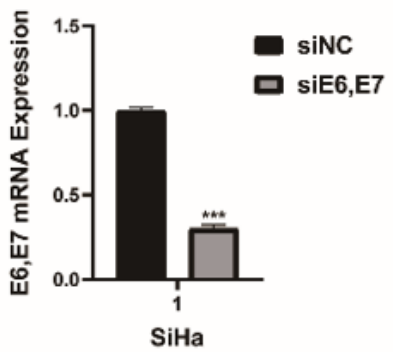

C

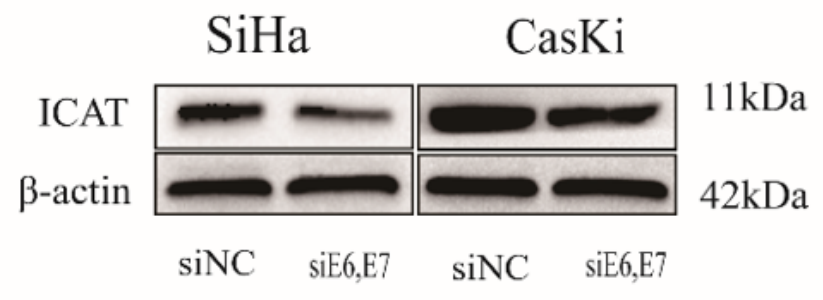

B

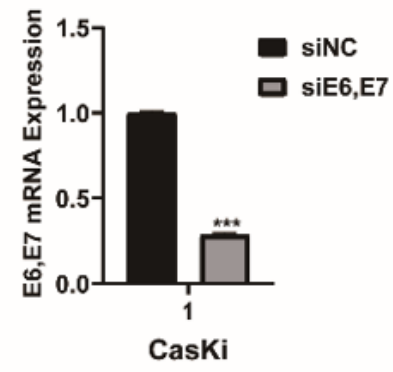

D
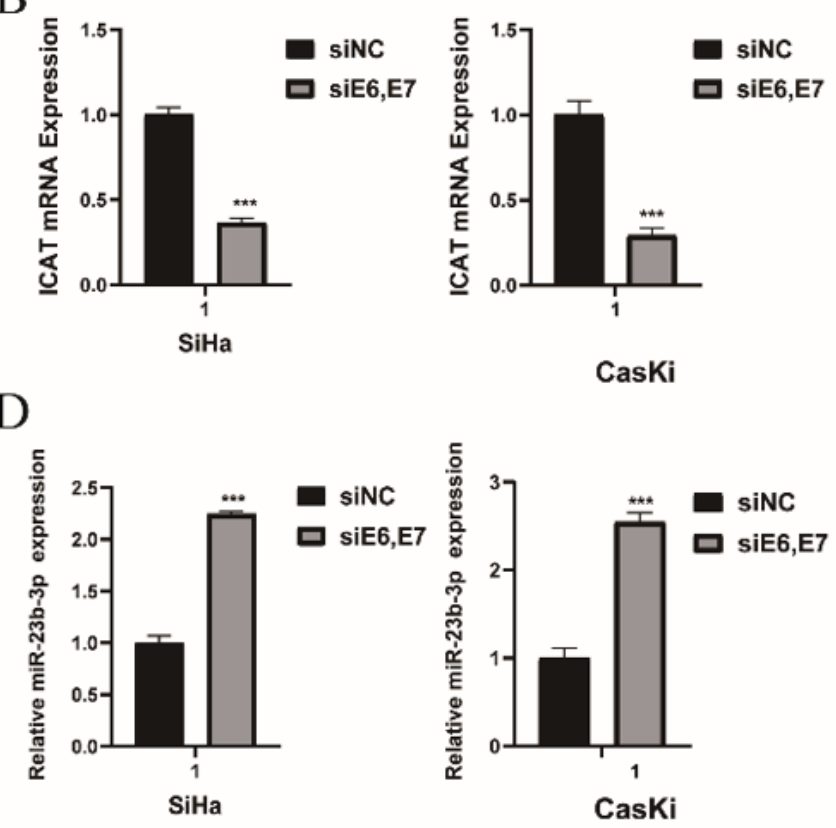

Figure 1

The effect of HPV16 E6, E7 on ICAT and miR-23b-2p expression in SiHa and CasKi cells. The SiHa and CasKi were transfected with HPV16 E6, E7 siRNA. (A) The knockdown efficiency of HPV 16 E6, E7 siRNA in cervical cells was analyzed by QRT-PCR. (B) The mRNA expression of ICAT in cervical cells was examined by qRT-PCR. (C) The protein expression of ICAT in cervical cells was determined by Western blot analysis. (D) The relative expression of miR-23b-3p in the cervical cells was detected by qRT-PCR. Data are expressed as mean $\pm S D(n=3)\left({ }^{*} P<0.05, * * P<0.01,{ }^{* \star} P<0.001\right.$, as compared with control group). 
A
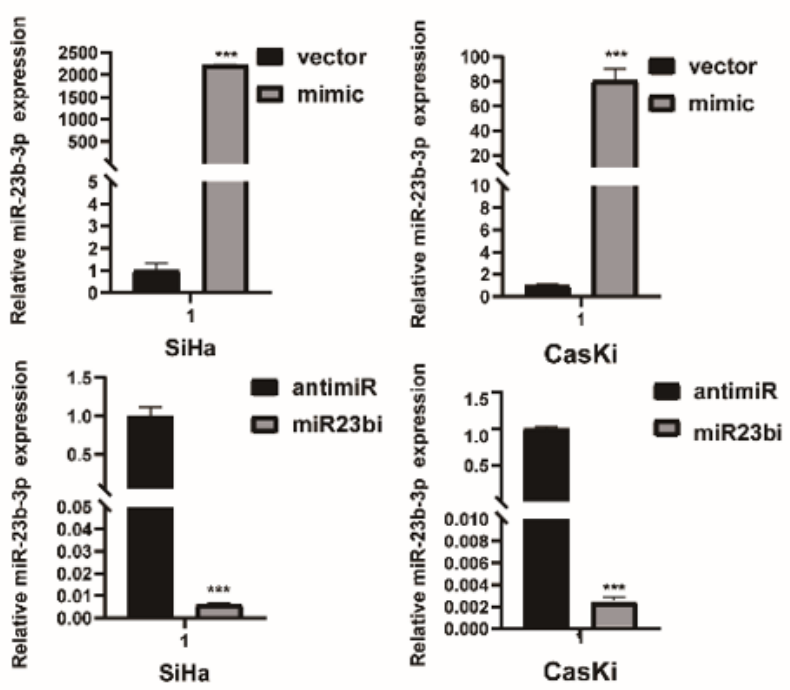

C

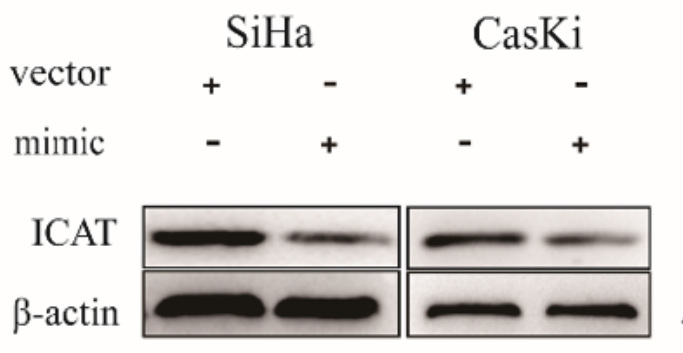

B
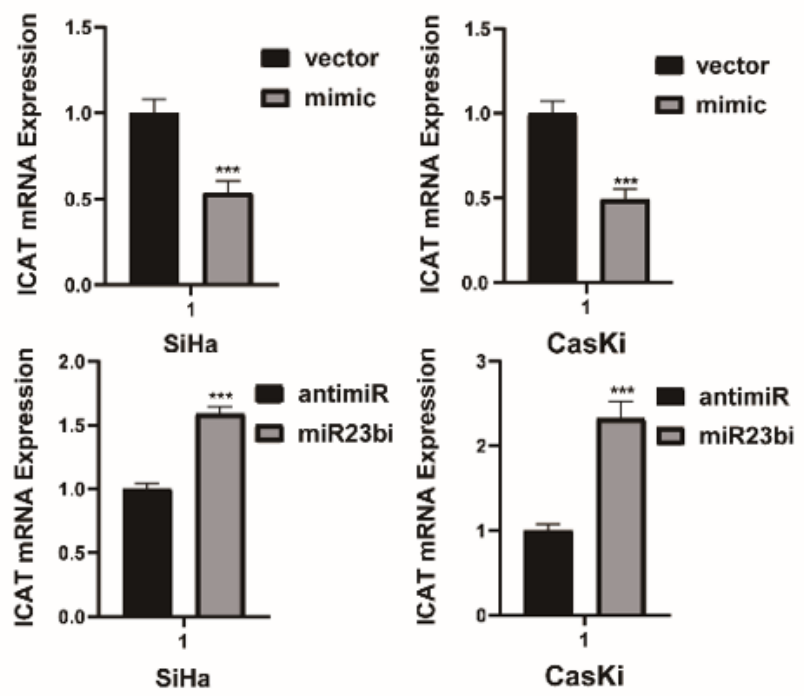

$11 \mathrm{kDa}$
$42 \mathrm{kDa}$

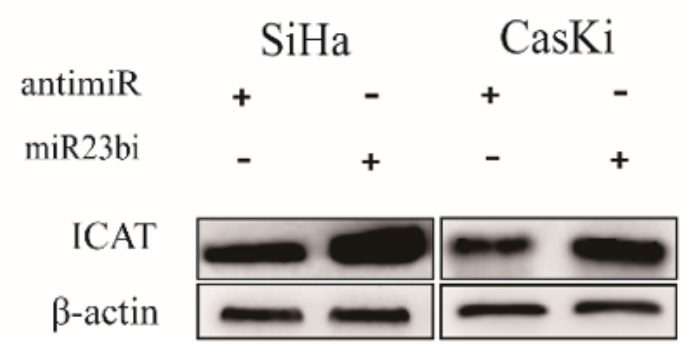

$11 \mathrm{kDa}$

$42 \mathrm{kDa}$

Figure 2

The effect of miR-23b-3p on ICAT expression in SiHa and CasKi cells. The SiHa and CasKi cells were transiently transfected with miR-23b-3p mimic, mimic NC (mimic or vector), anti-miR-23b-3p inhibitor, and anti-miR-23b-3p NC (miR23bi or antimiR) for 24h. (A, B) The expressions of miR-23-3p and ICAT mRNA were analyzed by qRT-PCR analysis. (C, D) ICAT protein expression was detected by Western blot analysis. Data are expressed as mean \pm SD $(n=3)$ ( ${ }^{2}<0.05$, $* \star$ $P<0.01$, ** $P<0.001$, as compared with control group). 
A

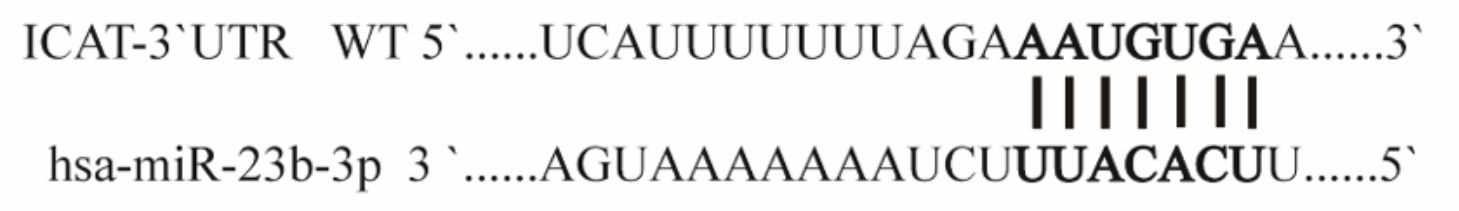
ICAT-3`UTR Mut 5' '......UCAUUUUUUUAGAGUAUAAGA.......3’

$\mathrm{B}$

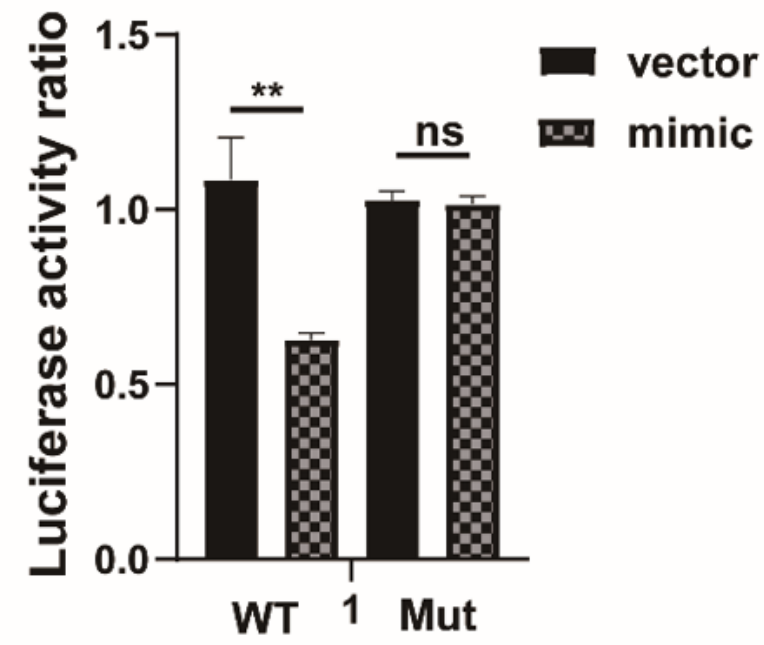

$\mathrm{C}$

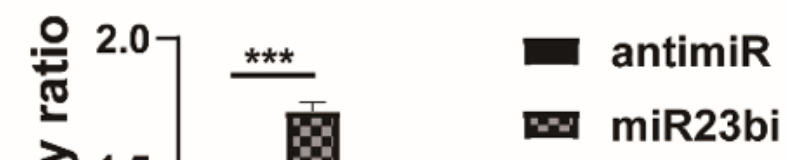

Figure 3

ICAT is a direct target of miR-23b-3p. (A) A graphical representation of the putative predicted the miR-23b-3p binding site in the ICAT mRNA 3 - UTR. (B) HEK293Tcellswereco - tranectedwithmiR - 23b-3pmimic, mimicNC (mimic or $\stackrel{\rightarrow}{\rightarrow} r)$, ICAT - 3-UTR Wt, and ICAT-3 -UTRMut. Luc if eraseactivitywasdeter min ed48hafter-tranectionbyluc if eraseassay. (C)HEK293Tcellswereco-tranectedwithanti-m. -UTR Wt, and ICAT-3'-UTR Mut. Luciferase activity was determined $48 \mathrm{~h}$ post-transfection by luciferase assay. UTR: untranslated region; WT: wild-type; Mut:

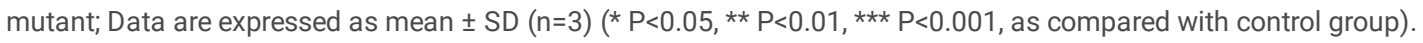


A

B

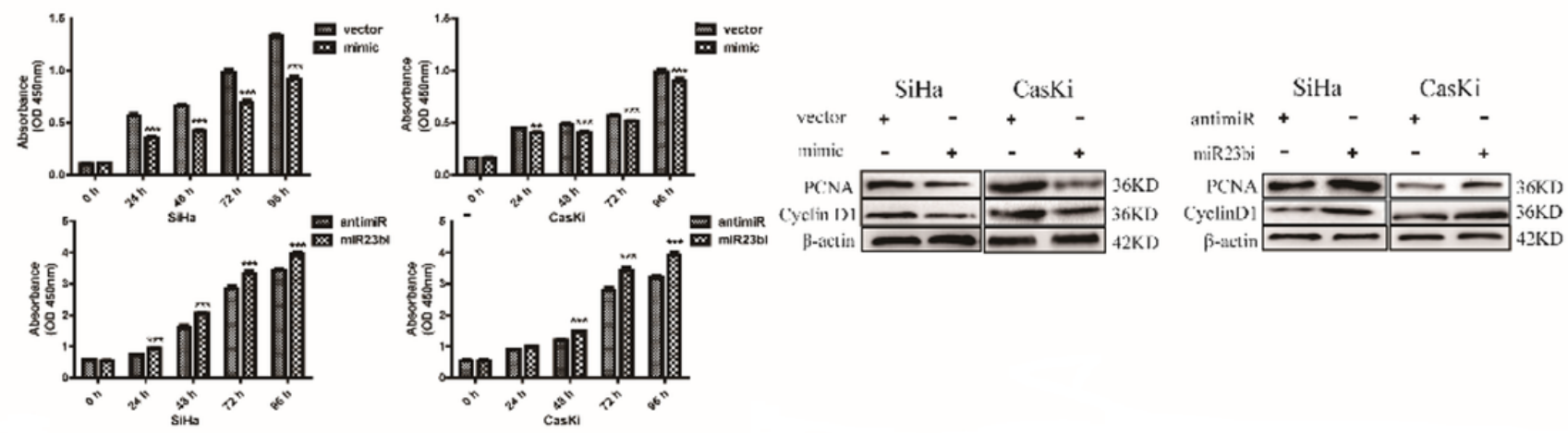

C
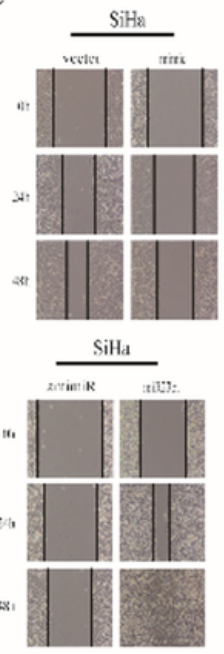
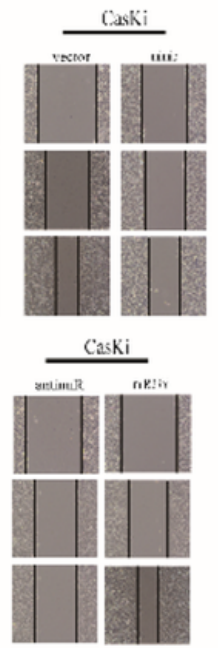

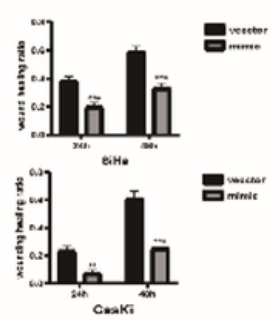

D
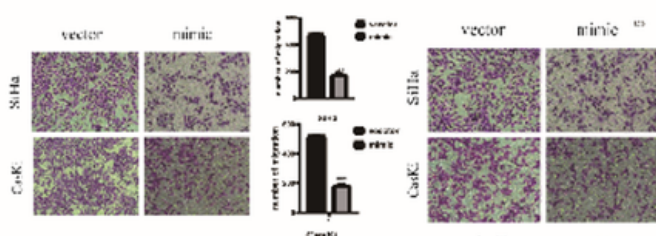

antimik nik233i
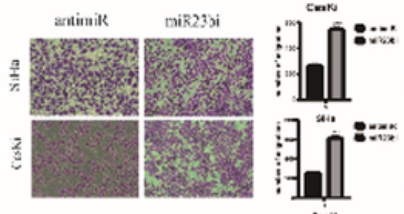

antivik

niR2:s:
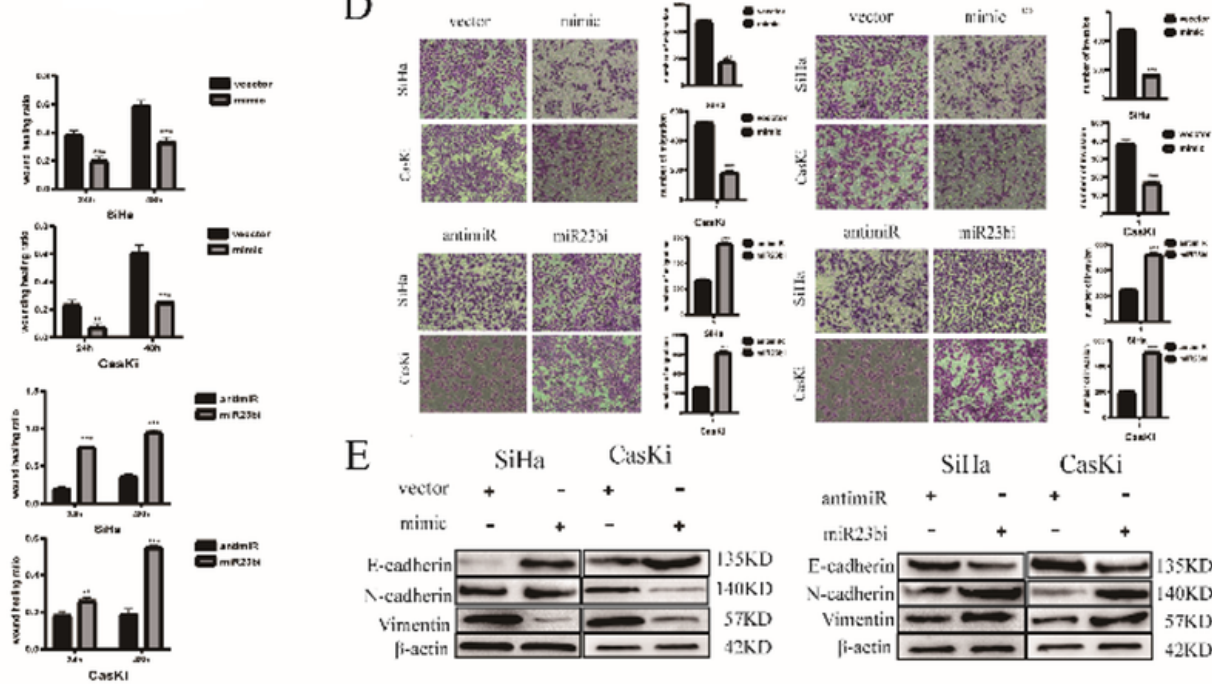

E
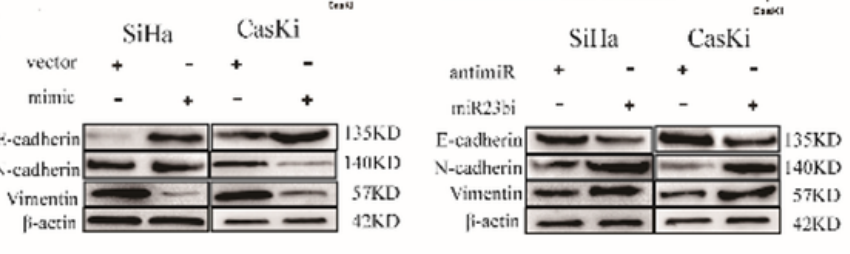

\section{Figure 4}

Effects of miR-23b-3p on the proliferation, migration, invasion and EMT of cervical cancer cells. The SiHa and CasKi cells were transiently transfected with miR-23b-3p mimic or mimic NC (mimic or vector) and anti-miR-23b-3p inhibitor or anti-miR-23b-3p NC (miR23bi or antimiR). (A) Cell proliferation was measured by CCK-8 assay. (B) PCNA and Cyclin D1 were detected by western blot analysis. (C) The migratory distance was assessed by wound healing assay. (D) The migration and invasion abilities were assessed by transwell assay. (E) The expressions of EMT-related protein markers (E-cadherin, N-cadherin and Vimentin) were detected by Western blot analysis. Data are expressed as mean $\pm S D(n=3)\left({ }^{*} P<0.05,{ }^{* *} P<0.01,{ }^{* \star *} P<0.001\right.$, as compared with control group). 
A

B

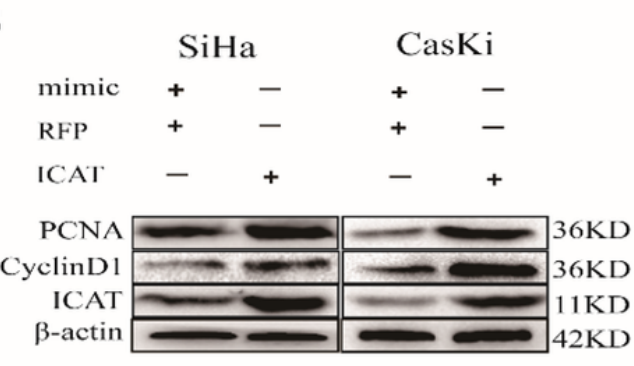

C
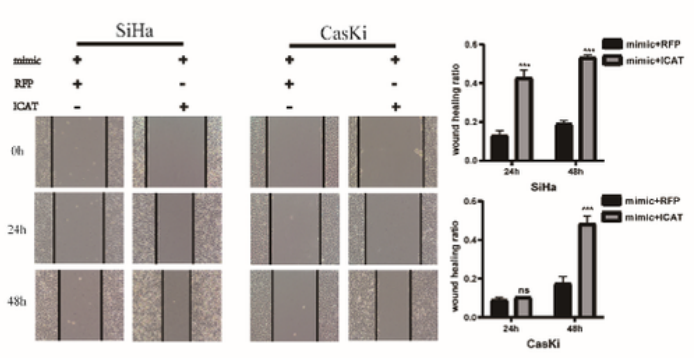

D

E
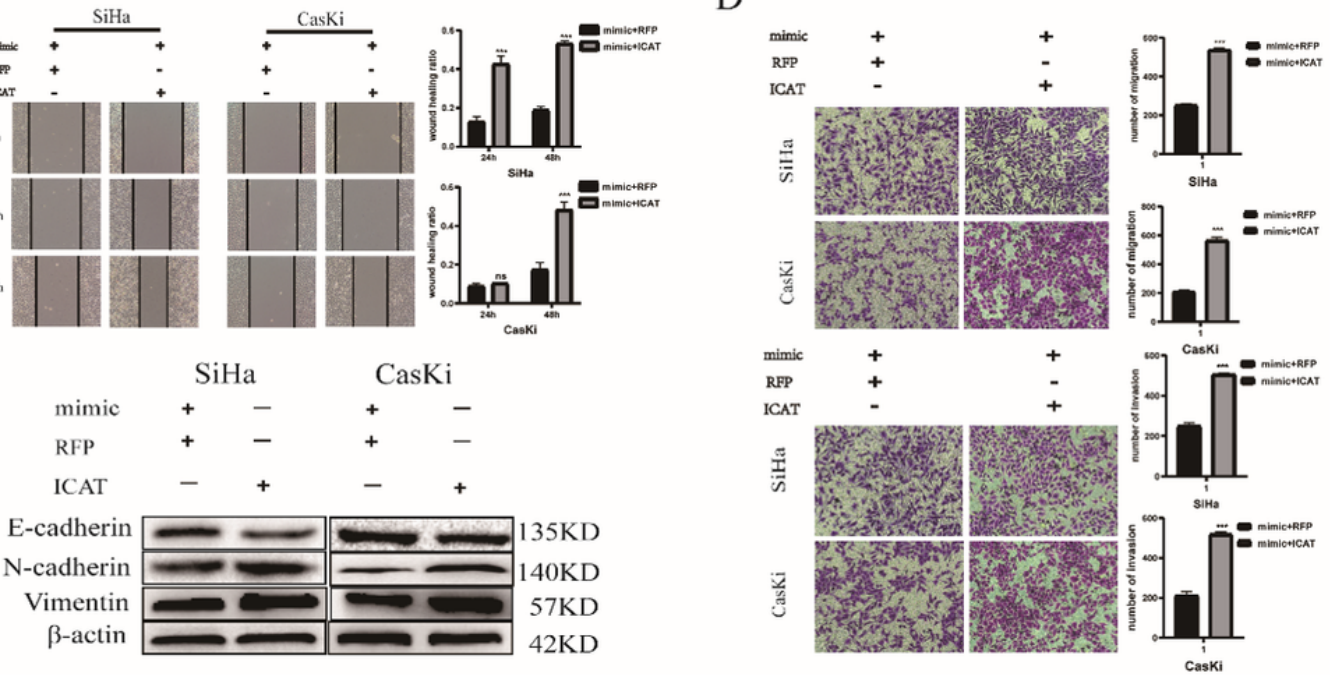

Figure 5

ICAT counteracts the effect of miR-23b-3p on cervical cancer cells. The SiHa and CasKi cells were co-transfected with miR-23b-3p mimic (mimic), AdICAT, and AdRFP. (A) Cell proliferation was measured by CCK-8 assay. (B) ICAT, PCNA, and Cyclin D1 were detected by western blot analysis. (C) The migratory distance was assessed by wound healing assay. (D) The migration and invasion abilities were assessed by transwell assay. (E) The expressions of EMT-related protein markers (E-cadherin, N-cadherin and Vimentin) were detected by Western blot analysis. Ad: adenovirus; Data are expressed as mean \pm SD ( $n=3)$ ( $P<0.05, \star \star *$ $\mathrm{P}<0.01$, ** $\mathrm{P}<0.001$, as compared with control group). 
A

B

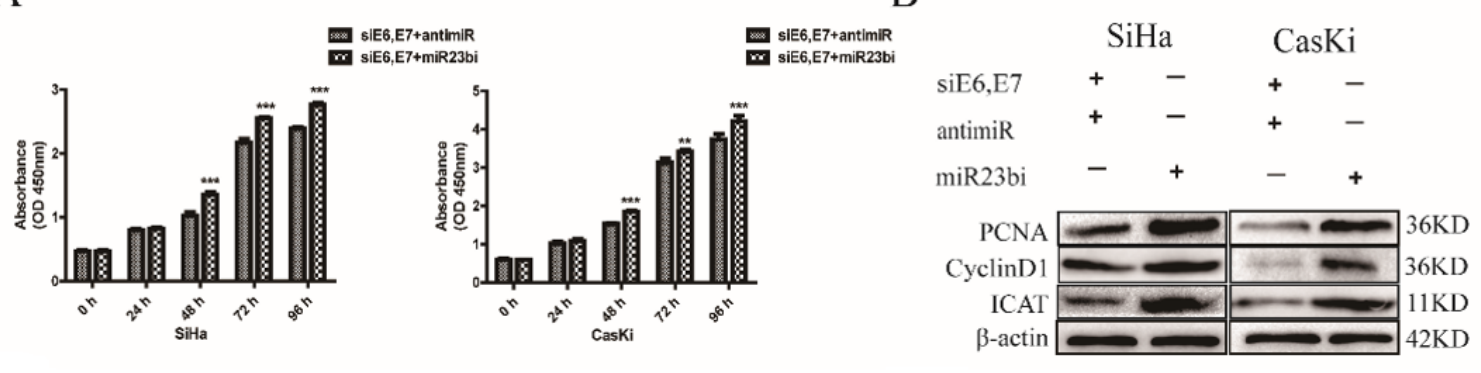

$\mathrm{C}$
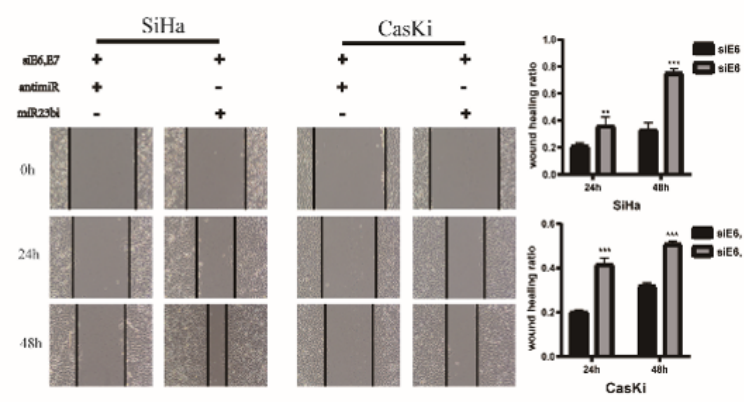

E

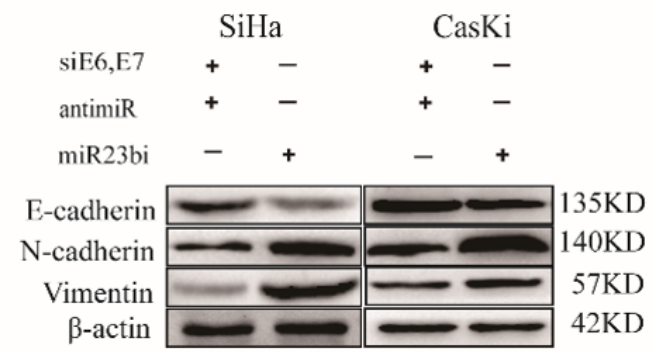

D
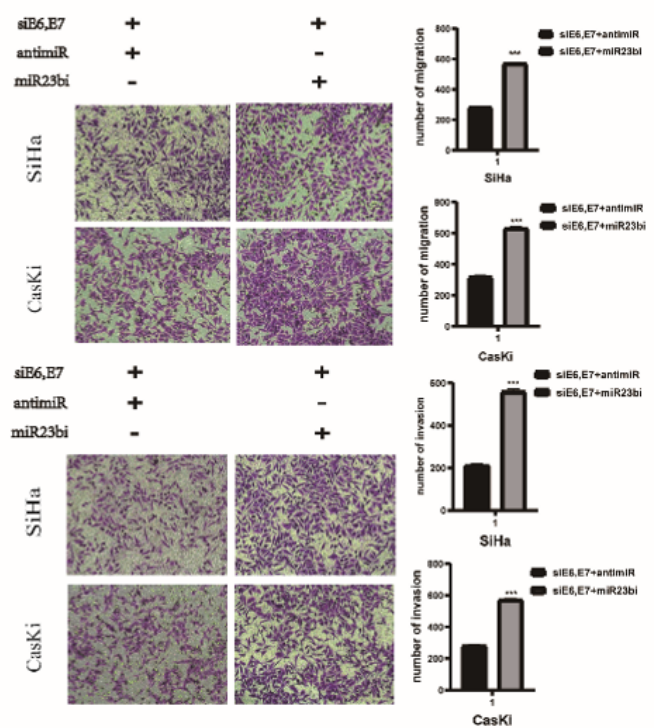

\section{Figure 6}

miR-23b-3p reverses the effect of HPV16 E6, E7 siRNA on cervical cancer cell. The SiHa and CasKi cells were co-transfected with HPV16 E6, E7 siRNA, antimiR-23b-3p inhibitor, amd anti-miR-23b-3p NC (miR23bi or antimiR). (A) Cell proliferation was measured by CCK-8 assay. (B) ICAT, PCNA and Cyclin D1 were detected by western blot analysis. (C) The migratory distance was assessed by wound healing assay. (D) The migration and invasion abilities were assessed by transwell assay. (E) The expressions of EMT-related protein markers (E-cadherin, N-cadherin and Vimentin) were detected by Western blot analysis. Data are expressed as mean $\pm S D(n=3)\left(* P<0.05\right.$, ${ }^{\star *} P<0.01$, *** $P<0.001$, as compared with control group). 


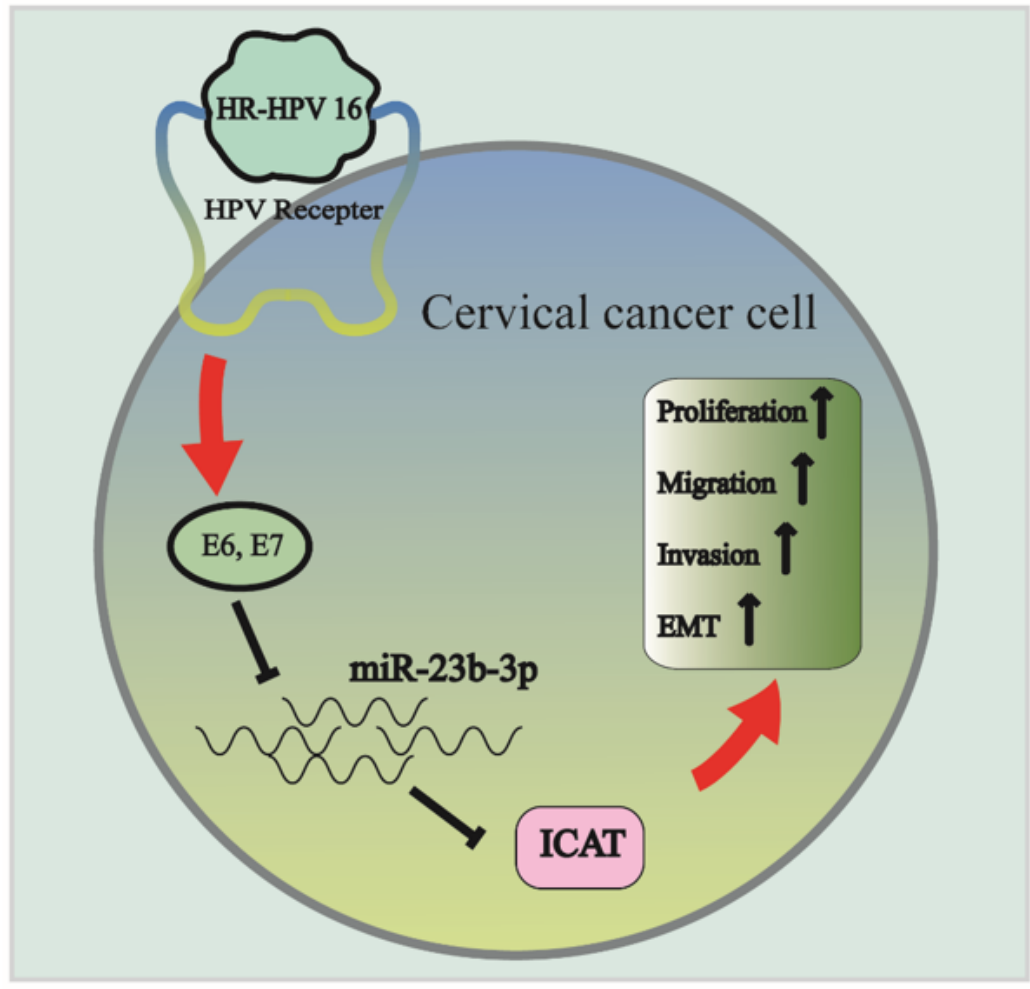

Figure 7

Model of HPV16 E6, E7/ miR-23b-3p/ ICAT axis in regulating the progression of cervical cancer. 